\title{
Michel Cabouret (1926-2005), In Memoriam
}

\section{François Carre}

\section{(2) OpenEdition}

\section{Journals}

Édition électronique

URL : http://journals.openedition.org/rge/1528

DOI : $10.4000 /$ rge. 1528

ISSN : 2108-6478

Éditeur

Association des géographes de l'Est

Édition imprimée

Date de publication : 1 juin 2006

ISSN : 0035-3213

Référence électronique

François Carre, « Michel Cabouret (1926-2005), In Memoriam », Revue Géographique de l'Est [En ligne], vol. 46 / 3-4 | 2006, mis en ligne le 16 décembre 2009, consulté le 15 septembre 2020. URL : http:// journals.openedition.org/rge/1528

Ce document a été généré automatiquement le 15 septembre 2020.

Tous droits réservés 


\title{
Michel Cabouret (1926-2005), In Memoriam
}

\author{
François Carre
}

Nécrologie parue dans " la Géographie », n 1522, septembre 2006, p. $96-99$ et publiée avec l'aimable autorisation du professeur Jean Bastié, directeur de la revue

1 Très affecté par la maladie puis la disparition de son épouse trois mois plus tôt, bien qu'il s'efforçât de ne point le laisser paraître, Michel Cabouret a été terrassé à son domicile de Woippy-lès-Metz au soir du 30 novembre 2005.

2 Ce Lorrain d'adoption était né à Paris où il avait fait ses premières études et fréquenté la Sorbonne dans l'immédiat après-guerre, jusqu'à l'obtention d'une licence d'histoire et de géographie, d'un diplôme d'études supérieures, puis du tout nouveau CAPES en 1952. Son affectation à Nancy et son mariage devaient décider de son enracinement en Lorraine et de son attachement à cette province où se déroula ensuite toute sa carrière universitaire de 1952 à 1995, et sur laquelle il publia des articles et notes de géographie historique et rurale.

3 Pas à pas il avait franchi les nombreuses étapes du cursus universitaire qui conduisaient, à cette époque, des collèges et lycées aux facultés, en expérimentant au passage tous les statuts d'enseignant : maître auxiliaire, professeur certifié de 1952 à 1959, professeur bi-admissible à l'agrégation, professeur agrégé en lycée de 1961 à 1965, assistant de faculté de 1965 à 1969, successivement au collège littéraire de Reims, alors rattaché à l'université de Nancy, puis à la faculté des lettres de Nancy même, maître assistant à Nancy de 1969 à 1971, chargé d'enseignement à l'université de Metz, enfin professeur de géographie humaine, économique et régionale à l'université de Metz de 1982 à sa retraite, prise en 1995, dans sa soixante dixième année.

4 Dans cette longue carrière une large place fut accordée à la pédagogie et aux activités d'enseignement, tâches dont Michel Cabouret s'est acquitté avec un grand dévouement. Dans l'enseignement supérieur il a toujours fait passer l'intérêt de ses étudiants avant les avantages personnels de carrière qu'apporte la recherche. Les étudiants de Metz se souviennent $\mathrm{du}$ soin qu'il apportait à la mise au point de ses cours de CAPES et d'agrégation et nombre d'entre eux reconnaissent qu'ils lui doivent leur réussite à l'un 
de ces concours. Jamais il ne s'est dérobé à la préparation des questions de géographie humaine ou régionale inscrites aux programmes, même quand elles portaient sur des régions qui ne lui étaient pas familières et qu'il n'avait parcourues qu'à travers les livres. À ce propos, il disait avec humour que, sans ses cours, il n'aurait jamais fait le tour du monde.

5 En effet, s'il avait bien participé, en compagnie de quelques anciens camarades d'études, à des missions de recherches en Asie occidentale et centrale (Turquie en 1953, puis Afghanistan pour trois mois en 1956), Michel Cabouret était un géographe plutôt casanier, ce que l'on peut expliquer par au moins deux raisons : une certaine phobie de l'avion et une fidélité sans faille aux pays de l'Europe du Nord qu'il avait choisis comme terrain d'étude dès sa jeunesse, - la première raison étant peut-être cause de la seconde -. Cette orientation régionale vers l'Europe du Nord découle de l'obtention, dès la fin de sa licence, de bourses de long séjour à l'université d'Oslo, 8 mois en 1949, 3 mois en 1950, mises à profit pour y préparer son mémoire de diplôme d'études supérieures soutenu en 1951.

6 Ce premier contact avec le monde scandinave se révéla déterminant pour toute sa vie de géographe. À Oslo il avait commencé à apprendre le norvégien qu'il finit par lire, écrire et parler couramment, ce qui lui donnait accès à la compréhension du suédois et $\mathrm{du}$ danois. Lorsqu'il envisagea la préparation d'une thèse de doctorat d'État en géographie pour accéder à l'enseignement supérieur, après avoir obtenu l'agrégation de géographie en 1961, son choix se porta spontanément sur la Scandinavie. Ses recherches sur «La vie pastorale dans les montagnes et les forêts de la péninsule scandinave » l'accaparèrent ensuite pendant dix neuf ans, jusqu'à la soutenance à la Sorbonne (Paris IV) en avril 1980 d'un mémoire de plus de 2800 pages. Ce faisant Michel Cabouret témoignait de sa fidélité à cette Europe du Nord qu'il avait découverte et choisie dans sa jeunesse et à sa Lorraine d'adoption, puisqu'il s'était adressé, pour encadrer ses travaux, à l'un de ses maîtres à l'université de Nancy au début des années 1960, le professeur Xavier de Planhol. Par conséquent, pendant plus de quinze ans, les voyages de Michel Cabouret furent « captifs » de la Scandinavie où il se rendait presque tous les ans en automobile ou en train et en bateau, avec une prédilection particulière pour ce dernier mode de transport.

7 Après la soutenance de sa thèse, loin de tourner cette page, Michel Cabouret continua de s'intéresser au monde scandinave. Il aimait venir s'y ressourcer le plus souvent possible, et pour une dernière fois encore en août 2005, mais par avion cette fois-ci pour s'épargner les fatigues d'un voyage en train. Sans abandonner la Norvège et la Suède, il avait cependant peu à peu élargi ses horizons à des pays voisins, à la Finlande d'abord, puis aux pays Baltes et notamment à l'Estonie, après l'éclatement de l'URSS. Aussi a-t-il pu consacrer l'essentiel de sa retraite à la rédaction de deux ouvrages de synthèse :

8 - l'irrigation des prés de fauche en Europe occidentale, centrale et septentrionale, essai de géographie historique, paru en 1999 , qui témoigne de son érudition et de sa culture historico-géographique, ce qui lui valut de recevoir le prix Eugène Gallois de la Société de Géographie (Paris) en 2000 ;

9 - la Finlande (2005, édit. Karthala), volume de géographie régionale qu'il eut encore la joie de voir paraître et pour lequel la Société de Géographie de Finlande lui décerna, un mois avant sa mort, la médaille Wilhelm Ramsay, tandis qu'en France la Société de 
Géographie le récompensait à nouveau, mais hélas à titre posthume, par le prix Conrad Malte Brun en 2006.

$10 \mathrm{Au}$ fil des ans, Michel Cabouret était devenu l'un des rares spécialistes français du monde scandinave dont il avait une connaissance exceptionnelle et sur lequel son érudition était rarement mise en défaut. La plupart des articles, notes et chapitres d'ouvrages collectifs qui jalonnent sa bibliographie, riche d'environ 150 titres, portent témoignage de cette familiarité étroite et profonde avec l'Europe du Nord. Ses collègues et amis savaient que l'on pouvait compter sur sa disponibilité et sa ponctualité quand on lui demandait une contribution dans ce domaine ou sur le thème de la géographie rurale historique. Mais, en retour, sa droiture et son intégrité lui interdisaient de transiger sur les exigences intellectuelles et de remettre des manuscrits trop hâtivement préparés et scientifiquement peu étayés. Ses écrits révèlent bien son souci méticuleux de l'exactitude et de l'exhaustivité qui se manifeste jusque dans son style. Ses longues phrases, souvent pleines de détours et riches en incises, que les amateurs de concision pouvaient juger pesantes et laborieuses, exprimaient cette volonté de ne rien laisser dans l'ombre.

Depuis longtemps les Scandinaves et les Finlandais avaient reconnu la compétence scientifique de Michel Cabouret et lui avaient manifesté leur estime. Plusieurs distinctions lui furent décernées: il était docteur honoris causa de la Faculté des Sciences sociales de l'Université d'Umea en Suède (1987), membre de l'Académie Royale Gustave-Adolphe d'Uppsala depuis octobre 1988, et membre d'honneur de la Société de Géographie de Finlande (1987). Ces honneurs qu'il avait obtenus à l'étranger sans les avoir sollicités, il les avait acceptés comme la juste récompense d'une vie de travail consacrée à ces pays du Nord, sans en tirer vanité et sans en faire étalage dans son propre pays. À en juger par sa modestie et sa simplicité, les mobiles de son activité scientifique appartenaient à un autre registre, celui qui tient habituellement les universitaires à l'écart de la notoriété tapageuse, la passion de la recherche désintéressée et le goût de la transmission du savoir. Cette passion l'aura habité jusqu'à ses derniers instants, puisque la mort l'a brutalement fauché alors qu'il venait d'entreprendre un nouvel ouvrage, sur la Norvège cette fois-ci. De façon symbolique, sa plume s'est immobilisée à jamais juste après qu'il eut écrit cet ultime mot, " Norvège ", auquel son nom restera attaché. 